\title{
Rheological Properties of Hybrid Hydrogels of Weakly Charged Polyelectrolytes Crosslinked by Aluminoxane Particles
}

\author{
S. S. Radchenko ", I. A. Novakov, Ph. S. Radchenko, E. V. Pis areva, A. S. Oze rin \\ Department of Analytical and Physical Chemistry and Phy sical Chemistry of Polymers, Volgo grad State Technical University, 400005, \\ Russian Federation
}

\begin{abstract}
The rheological behavior of hybrid hydrogels of weakly charged polyelectrolytes with aluminoxane particles (APs) formed by noncovalent interactions has been studied. This interaction observed for the polyelectrolyte Praestol-2500 is the most pronounced and is due to strong electrostatic noncovalent bonds between positively charged APs and anionic groups of the copolymer. By the methods of capillary, rotational, and vibrational rheo metry, it has been ascertained that two processes compete: the compacting of coils of macromolecules and their crosslinking through APs. The latter process dominates at relatively high concentrations of reagents, and depends on ratios between polymer units and APs and the presence of a low-molecular-mass indifferent electrolyte $(\mathrm{NaCl})$. Formed hybrid hydrogels relate to physical gels with the unstable nature of the network of physical bonds, and under the action of shear stress demonstrate "Weiss enberg effect." The method of dynamic mechanical analysis with the use of a micro-Fourier rheometer has shown that the ratio storage modulus - loss modulus depends on the amount of AP sol and its ratio to the quantity of copolymer in the initial mixture. A scheme for spatial structure hybrid hydrogels has been suggested.
\end{abstract}

Keywords Hybrid Hydrogels, Weakly Charged Polyelectrolytes, Aluminoxane Particles

\section{Introduction}

At present, the creation of composite materials is characterized by the use of nanodisperse materials of both inorganic and organic nature. Such composites are often referred to as hybrid nanomaterials.[1] This term is often related to purely inorganic nanocrystals and semiconductors, [2],[3] organic nanocomposites,[4],[5] and mixed disperse systems. In the latter case, it is assumed that the composite includes metal or metal oxide (hydroxide) particles as the disperse phase, whereas a polymer or its solution plays the role of the continuous phase.[6-10]

Polymeric organo-inorganic composites belong to the promising class of hybrid materials due to a unique combination of magnetic, catalytic, nonlinear optical, and sensoric properties of inorganic nanoparticles with a set of properties of the polymeric matrix and its ability to stabilize inorganic clusters dispersed in it.

There are a lot of methods for obtaining nano-objects and their composites.[11-14] The interaction of linear organ ic macromolecules with aqueous dispersions of charged

* Corresponding author:

radchenko@vstu.ru (S. S. Radchenko)

Published online at http://journal.sapub.org/cmaterials

Copyright (C) 2012 Scientific \& Academic Publishing. All Rights Reserved nanoparticles (hydrosols) may be regarded as a possible approach to the production of organo-inorganic nanocomposites.

This method looks attractive because (i) it makes possible the controllable arrangement of nanoparticles in a polymeric matrix, that is, via its reaction centers and (ii) the so-called polymer-collo id complexes (PCCs) (for brevity sake, named polycomplexes) that are formed in this case are generated spontaneously due to noncovalent interactions.[15-17] The formation of polyco mplexes may proceed during the sol-gel synthesis of nanoparticles in the presence of polymers [18-20] or through the simplest method-via the blending of a sol with a polymer solution.[15],[21-23] Exactly this method was used to prepare polycomplexes of some types of water-soluble poly mers with alumino xane particles (APs) in the sols of superbase polyaluminum hydroxychloride (PAHC).[24-26] These complexes represent the colloidal dispersion of APs formed during the hydrolysis of some salts of aluminum as a result of the polycondensation of aluminum aqua hydroxo complexes and are distinguished by high aggregate stability at a wide range of concentrations and temperatures.[27] On the basis of small-angle X-ray scattering studies, APs may be related to physical fractals having an internal self-similar structure with a fractal dimensionality $\mathrm{d}_{\mathrm{f}}=1.0$ and a radius of gyration $\mathrm{r}_{\mathrm{g}}=1.6$ $\mathrm{nm}$.[28] Various kinds of noncovalent interactions are 
typical for APs. As a consequence, they may form polycomplexes both with nonionogenic polyacrylamide (PAA)[24],[26] and with its cation-active[29] and anion-active[30] copolymers. However, soluble polycomplexes are obtained only at definite ratios of APs to functional groups of copolymers. Otherwise, insoluble polycomplexes are formed if unlikely charged reagents are used or polycomplexes are not formed at all because of large forces of repulsion of likely charged reagents. Polycomplexes of PAHC sols with polyacrylamide show promise for practice as regulators of the stability of disperse systems, for example, in the processes of purification of natural water and sewages,[31-33] in enhanced oil recovery techniques [34],[35] and as environmentally friendly binding agents for disperse materials.[36] In all the above-listed cases, hybrid polycomplexes are used in the form of aqueous suspensions or gels, that is, fluid systems. At the same time, no data are available on the rheological behavior of these new systems. For most real liquids, irreversible deformations, which appear during flow, are characterized by the complicated dependence of different factors, and their rheological behavior is often in the intermediate region between the liquid and solid. This feature is especially pronounced for high-molecular-mass polymers, aqueous solutions of which possess viscoelastic properties. The pattern of the flow of polymer solutions often determines the possibility of their processing and application. Therefore, the rheological study of such systems is of great importance for practice. Moreover, in addition to its main direct purpose-determination of the rheological characteristic of fluid systems-rheology may serve as a structural method that relates rheological parameters and structural changes in a deformed system. [37]

The goal of this work was to study the rheological behavior of aqueous solutions and hydrogels of polymer-colloid complexes of APs with weakly charged polyelectrolytes. As far as particles in a polycomplex occur in the chemically bound via noncovalent bonds state rather than in the inert form, they naturally affect the conformational state of polymer mac ro molecules. Therefore, to compare the rheological properties of a polymer and its polycomplex, aqueous solutions of initial polymers were also investigated. As it is known, for polymer solutions, the regions of diluted and semidiluted solutions exist outside the crossover region, where the elastic properties of polymer liquids begin to manifest themselves. Therefore, capillary and rotational viscometry and the method of dynamic mechanical analysis with the use of a micro-Fourier rheometer were applied as investigation procedures.

\section{Experimental}

\subsection{Initial Materials}

A dispersion of APs was prepared froman alu minu malloy in the form of an aqueous sol containing $13.5 \mathrm{wt} \% \mathrm{Al}^{3+}$ and with the atomic ratio $\mathrm{Cl}^{-} / \mathrm{Al}^{3+}=0.45$. The technique of preparation and the characteristics of the sol as well as the dimensional and fractal characteristics of APs were described in[27],[28].

As weakly charged polyelectrolytes, commercial samples with characteristics listed in Table 1 were employed; they were used without any additional purification.

An aqueous solution of polyacrylamide was prepared via the free-rad ical poly merization of acrylamide $(99.9 \%$; Alfa Aesar, USA) in an aqueous solution ( $3 \mathrm{wt} \%$ acryla mide) in the presence of potassium persulfate ( $97 \%$; Alfa Aesar, USA) at a temperature of $60^{\circ} \mathrm{C}$ and was used without additional purification.

Sodium chloride of special purity grade $(99.99 \%$; Alfa Aesar, USA) was used. In all experiments, twice-distilled water was used. Polycomplexes were prepared by mixing aqueous solutions of polyelectrolytes of different concentrations with preset quantities of the AP sol. After shaking in a shaker, aqueous dispersions (gels) were allowed to stand at room temperature for a day.

\subsection{Rheological Investigations}

Viscometric measurements were performed by means of a Brookfield DV-II+Pro (USA) rotary viscometer equipped with a UL (Ver. Ro $4 \mathrm{~s} / \mathrm{s}$ ) adapter and a thermostated cell. Experimental data were processed with the use of a DVLOADER authorized program.

The yield stress was determined on a YR-1 special-purpose rheometer equipped with a set of V-71-73 vane spindles. The measurement data were processed by means of a YR-1-4A Y authorized program.

Viscoelastic properties were investigated on an MFR-2000 micro-Fourier rheometer (GBS, Australia). Measurements were performed in the frequency interval $0-100 \mathrm{~Hz}$ with a step of $1 \mathrm{~Hz}$ at a temperature of $25^{\circ} \mathrm{C}$. All variable parameters and calculations of measurable characteristics were enclosed in an MFR-2100 Ver. 1.1b4 authorized program.

\section{Results and Discussion}

Polymer-colloid complexes of APs with water-soluble polymers are thermodynamically stable compounds with a variable composition in the case of soluble polycomplexes[26] or with a characteristic composition when polycomplexes are insoluble.[38] As was shown previously, polycomplexes may be soluble either if nonionogenic polyelectrolytes[25] or weakly charged polyelectrolytes [30] are used. In both cases, polycomplexes are transparent homogeneous disperse systems with specific physicochemical features peculiar to every component. One of them is the aggregative stability of each of the dispersion phases. The specific feature of the given systemis that the sol of PAHC is a known regulator of the stability of dispersion systems, whereas a water-soluble poly mer is often used as a flocculant in the separation of dispersion systems. The rheological behavior of each of the reagents may change in 
the presence of other reagents. Moreover, both reagents are polyelectrolytes; hence, they are influenced by the ionic strength of the solution. The superposition of such specific features on the properties of interacting reagents may cause inadequate dependences. Therefore, both interacting disperse systems-AP sols and aqueous solutions of polyelectrolytes - as well as polycomplexes, the products of their interaction, were investigated separately.

\subsection{Investigation of Rhe ological Characteristics of the Sol of Aluminoxane Particles}

Figure 1 presents the experimental data on the kinematic viscosity of the AP dispersion measured at various concentrations of the disperse phase $\left(\mathrm{Al}^{3+}\right)$, and demonstrates the effect of a low-molecular-mass indifferent electrolyte $(\mathrm{NaCl})$ on the aggregative stability of the sol.
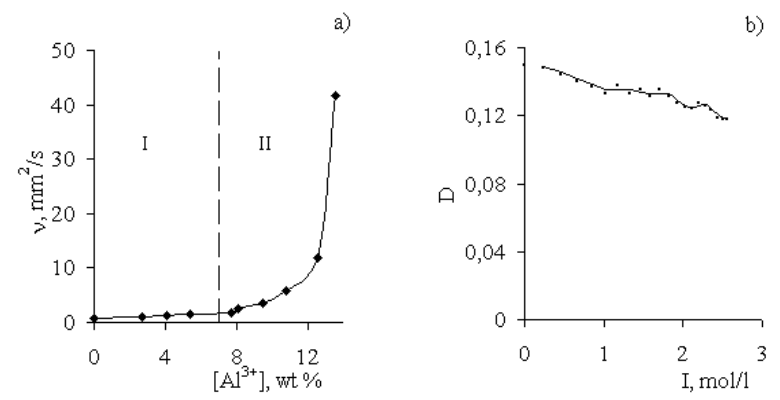

Figure 1. Dependence of (a) kinematic viscosity (v[nu]) of AP sol on the concentration of disperse phase $\left[\mathrm{AP}^{\beta+}\right]$ and (b) optical density of sol $\left(\left[\mathrm{Al}^{3+}\right]=\right.$ $13.5 \mathrm{wt} \%)$ on the ionic strength of solution $(\mathrm{I}, \mathrm{NaCl})$

The curve for the kinematic viscosity of the sol is composed of two regions: region I, where there is the linear dependence of viscosity on the concentration of the disperse phase corresponding to the free-disperse state of the system, and region II, where there is the exponential dependence conforming to the transition of the colloid system to the bound-dispersion state-gel.
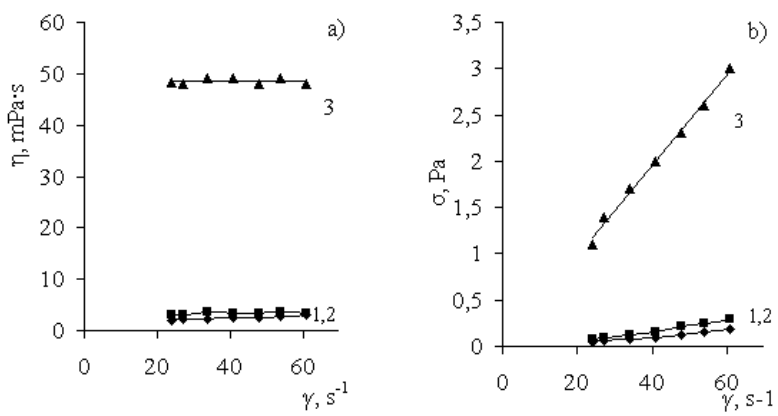

Figure 2. Curves for (a) viscosity and (b) flow of AP sol at different concentrations of disperse phase ( $\mathrm{AP}^{3+}$, wt\%): (1) 4.5, (2) 6.8, and (3) 13.5. T $=30^{\circ} \mathrm{C}$

However, the flow of dispersion in the annular gap of the rotary viscometer is typical for Newtonian liquids; that is, viscosity is independent of the shear rate within the whole concentration range, and a change in shear stress follows a linear pattern (Figure 2).
The PAHC gel (the sol in a concentrated state containing $13.5 \mathrm{wt} \% \mathrm{Al}^{3+}$ ) features a peculiar behavior in the presence of $\mathrm{NaCl}$. Upon the addition of $\mathrm{NaCl}$ in the form of a concentrated aqueous solution, the aggregative stability of the dispersion is preserved (Figure 1b); however, this operation leads to an adequate decrease in the dynamic viscosity of the disperse system. On the whole, the flow of this system remains similar to the flow of a Newtonian flu id (Figure 3).
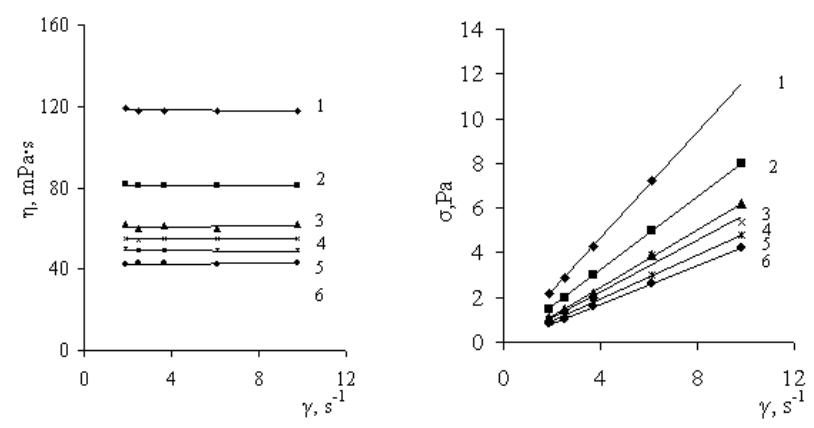

Figure 3. Curves forviscosity $(\eta)$ and flow $(\sigma)$ of PAHC gel $\left(\left[\mathrm{Al}^{3+}\right]=13.5\right.$ wt \%) upon the addition of $\mathrm{NaCl}$ solution with concentrations (wt\%): (1) 0 , (2) 0.9, , (3) 1.8, , (4) 2.7, (5) 3.6 , and (6) 4.5

However, when $\mathrm{NaCl}$ is added in the dry state, the fluctuation formation of particles, nuclei of the new phase, occurs, and the coagulation structure appears to be accompanied by the transition of the whole disperse system into a solid-like state without evolution of the dispersion medium-water. This case was realized through the preparation of the solid PAHC.[39],[40]

\subsection{Rhe ological Properties of Aque ous Solutions of Weakly Charged Pol yelectrolytes and Their Complexes with Aluminoxane Particles}

The behavior of polyelectrolytes in aqueous solutions is determined by the conformational state of macromolecules and their concentrations. In this study, we selected three kinds of water-soluble polymers: nonionogenic polyacrylamide, weakly anionic Praestol-2500, and weakly cationic Praestol-611 BC. Some of their characteristics are given in[29],[34]. It should be noted that the characteristics of commercial samples are tentative. Thus, Praestol-2500 relates to nonionogenic polymers. However, as follows from potentiometric analysis, $1.2 \mathrm{~mol} \% \mathrm{COOH}$ groups are contained in Praestol-2500. Hence, this compound may be related to weakly anionic poly mers that form polycomplexes similarly to weakly charged copolymers of acryla mide with acrylic acid. [30] This is also the reason why polyacrylamide prepared under laboratory conditions via the free radical polymerization of reactive acrylamide was used as a nonionogenic polymer. In this case, its molecular mass was significantly smaller than those of commercial Praestol-2500 and Praestol-611 BC. As evidenced by potentiometric analysis, this polymer was free of $\mathrm{COOH}$ groups.

Soluble polymer-colloid complexes of APs with water-soluble polymers are thermodynamically stable 
compounds with variable compositions. Their behavior in aqueous solutions should evidently differ from the behavior of corresponding linear polymers. In the case of strongly diluted solutions, the intramolecular interaction of macromolecules with APs accompanied by the compaction of macromolecules may occur. As is known, under thermodynamically favorable conditions, with an increase in the polymer concentration, a network of topological entanglements appears between separate chains of mac ro molecules.[41] The concentration of crossover $C^{*}$ is a characteristic value of this state (Table 1).

Table 1. Properties of water-soluble polymers in $10 \%$ solution of $\mathrm{NaCl}$

\begin{tabular}{|c|c|c|c|c|}
\hline Polymer type & {$[\eta]$} & Charge & $\begin{array}{c}\text { Molecular } \\
\text { mass } \times 10^{6}\end{array}$ & $\begin{array}{c}\mathrm{C}^{*}, \\
\mathrm{dl} / \mathrm{g}\end{array}$ \\
\hline Polyacrylamide & 4.54 & 0.00 & $\sim 1.29$ & 0.22 \\
\hline Praestol-2500 & 17.50 & -1.20 & $\sim 14.01$ & 0.06 \\
\hline Praestol-611 BC & 7.54 & +6.52 & $\sim 6.01$ & 0.13 \\
\hline
\end{tabular}

The rheological behavior of aqueous solutions of the polymers under study should be determined by concentration intervals. As was shown in ([29],[30]), in the region of diluted aqueous solutions $(0.005-0.05 \mathrm{~g} / \mathrm{dl})$, there is the linear dependence of specific viscosity on the polymer concentration. In this case, the polyelectrolyte effect is dismissed due to the presence of $\mathrm{NaCl}$ in solution. However, the peculiarity of this system is that during the formation of polycomplexes, the sol of PAHC is added to the aqueous solution of the polyelectrolyte. This sol is a dispersion of APs carrying a positive surface charge and surrounded by $\mathrm{Cl}^{-}$ counterions; that is, the sol of PAHC is likewise an electrolyte. The calculation of the ionic strength of PAHC solutions is impossible because the charge and number of ions governing the potential on the surface of APs as well as the distribution of $\mathrm{Cl}^{-}$counterions between the dense and diffuse portions of the electric double layer are unknown. However, with consideration for high aggregative and sedimentation stability of PAHC sols,[27] it may be suggested that the charge of a colloid particle is rather high.

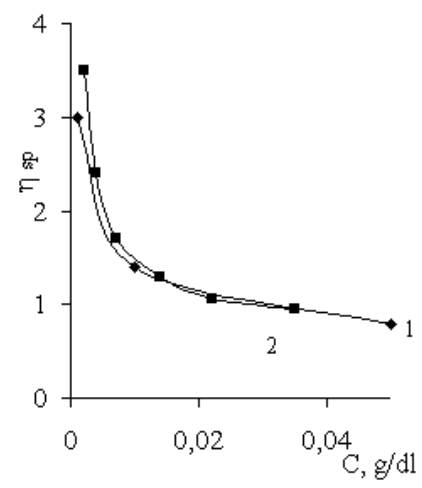

Figure 4. Dependence of specific viscosity of Praestol-611 BC solution on the nature and concentration of electrolytes: (1) $\mathrm{NaCl}$ and (2) PAHC. $\mathrm{C}_{\text {Praesto161 1 BC }}=0.05 \mathrm{~g} / \mathrm{dl}$

Figure 4 demonstrates a change in the specific viscosity of the aqueous solution of Praestol-611 BC upon the addition of
$\mathrm{NaCl}$ and the sol of APs at equal concentrations. The concentration of the polymer in solution is lower than the crossover concentration.

The above data show that the effects of both salts are almost the same and are accompanied by the compaction of macromolecular coils. In the case of $\mathrm{NaCl}$, compaction is caused by the shielding of charged functional groups in the polymer by counterions of the low-molecular-mass salt.[42] The degree of such a shielding is determined by the nature of counterions and the charge of the functional group of the polymer.

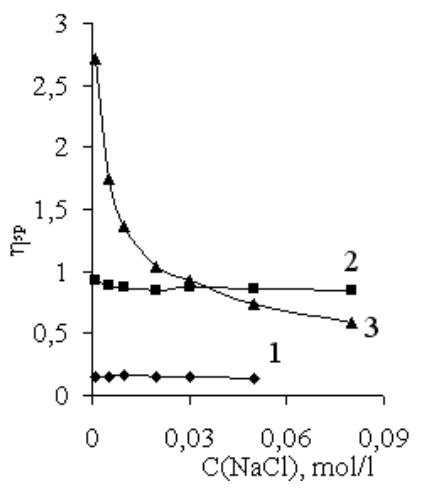

Figure 5. Dependence of specific viscosity of diluted solutions of polymers $(0.05 \mathrm{~g} / \mathrm{dl})$ on the concentration of $\mathrm{NaCl}$ in solution (mol/l): (1) polyacrylamide, (2) Praestol-2500, and (3) Praestol-61 1 BC

As is seen from Figure 5, for nonionogenic PAA, the presence of $\mathrm{NaCl}$ in solution exerts almost no effect on the value of specific viscosity. To a small extent, this phenomenon is observed for the solution of low-anionic Praestol-2500 and sharply manifests itself for Praestol-611 BC polycationite.

This phenomenon is probably related to a marked increase in the concentration of $\mathrm{Cl}^{-}$ions in solution of the polymer having counterions of the same type as Praestol-611 BC, which contains dis sociating functional $\mathrm{N}^{+}\left(\mathrm{CH}_{3}\right)_{3} \mathrm{Cl}^{-}$groups. As for the influence of the AP sol on a decrease in the specific viscosity of the Praestol-611 BC solution, two causes may be suggested. The first cause is that the ionic strength of the solution changes due to the dissociation of the high-basicity aluminum salt accompanied by the evolution of $\mathrm{Cl}^{-}$ions into the solution. The second cause is related to the intramolecular interaction of APs with polymer macromolecules, which is accompanied by the compaction of macromolecular coils. As can be seen from Figure 5, a decay in the specific viscosity of the solution takes place until a certain amount of introduced $\mathrm{NaCl}$ is achieved and further decrease in viscosity upon introduction of the AP sol into the solution of the polymer should be indicative of the formation of the polycomplex, as was shown for the diluted solutions of these polymers.[26],[29] Another rheological behavior should be observed for semiconcentrated solutions of polymers, where polymer coils begin to overlap.[44] In the transition state, which correlates with the so-called crossover concentration $\mathrm{C}^{*}$, the intermolecular interaction of APs with two or a greater amount of macro molecules may occur. However, at the macroscopic level, the related 
structuring of a polymer solution man ifests itself at poly mer concentrations significantly higher than $\mathrm{C}^{*}$. For disperse systems similar to the system under consideration (aqueous solution of polymer-sol of inorganic partic les), the deviation of the concentration dependence of viscosity from the linear pattern (the Einstein law) provides evidence for this phenomenon.[43],[44] In fact, the capillary v is cometry study of mixed solutions of polymer mixtures with APs showed that the deviation of the concentration dependence of viscosity on the concentration of the solution from the linear pattern begins at a polymer concentration almost twice higher than $C^{*}$ (Figure 6).

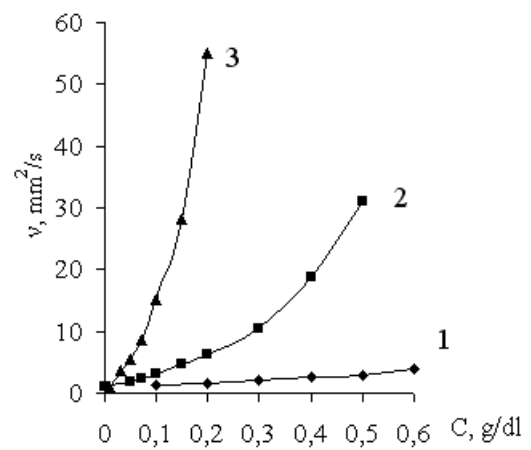

Figure 6. Kinematic viscosity $(v)$ of aqueous solutions of polycomplexes versus concentration of polymers (C): (1) PAA, (2) Praestol-2500, and (3) Praestol-611 BC

Therefore, the rheological studies of semiconcentrated solutions of polymers and their mixtures with the AP sol were conducted at polymer concentrations above $0.3 \mathrm{~g} / \mathrm{dl}$. The main goal of this study was to compare the rheological behavior of polymers and their polycomplexes; therefore, it was necessary to ensure equal conditions for rheometry measurements and to use the same measuring system. In this work, a Searle rheometer equipped with a UL-1 adapter with coaxial cylinders was used. This adapter with a thermostated jacket was rigidly fastened to the body of the viscometer, thereby providing the reproduction of equal experimental parameters (depth of mergence of spindle, eccentricity, and gap in cylinders) and minimizing the impact of the so-called apparatus effects on the results of measurements.[37]

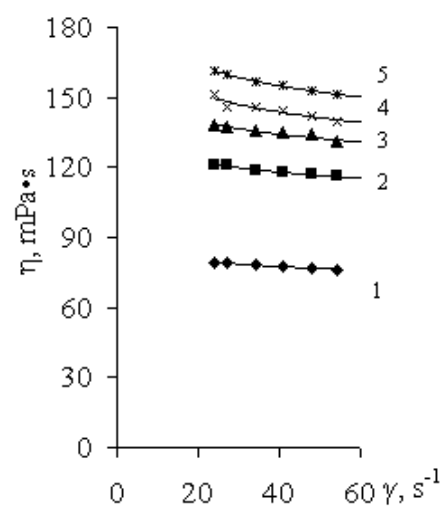

Figure 7. Curves for viscosity of solutions of (1) PAA $(2 \mathrm{~g} / \mathrm{dl})$ and its PCCs at various ratios (PAA unit: $\mathrm{AP}^{3+}(\mathrm{mol} / \mathrm{mol})$ ): (2) 1:0.5, (3) 1:1, (4) 1:2, and (5) $1: 4$

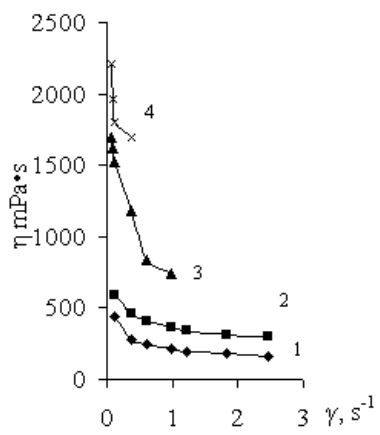

Figure 8. Curves for viscosity of solutions (1) of Pr-2500 (0.6 g/dl) and its polycomplexes with AP at various ratios (Pr-2500 unit: $\mathrm{Al}^{3+}$ (mol/mol)): (2) $1: 0.1$, (3) $1: 0.25$, and (4) $1: 0.37$

Figures 7 and 8 plot dynamic viscosity against shear rate for solutions of PAA and Pr-2500 polycomplexes with AP at various molar ratios of reagents.

As follows from these data, the pattern of curves is indicative of the viscoelastic properties of polymer fluids. Note that with an increase in the amount of APs in the polycomplexes, the contribution of elastic properties, especially in the case of $\operatorname{Pr}-2500$, noticeably grows. This suggests the occurrence of structuring processes in the polymer solution upon introduction of the AP sol.

Similar experiments were conducted with Pr-611. Taking into account its dependence on $\mathrm{NaCl}$, the experiments were performed in aqueous and aqueous-salt solutions. The quantitative results of these rheometric measurements are shown in Figure 9 in the form of columnar diagrams.

The above rheometric data show that the addition of both $\mathrm{NaCl}$ and AP sol to the aqueous solution of Pr-611 leads to a strong drop in the viscosity of the solution and in the shear stress (Figure 9a).
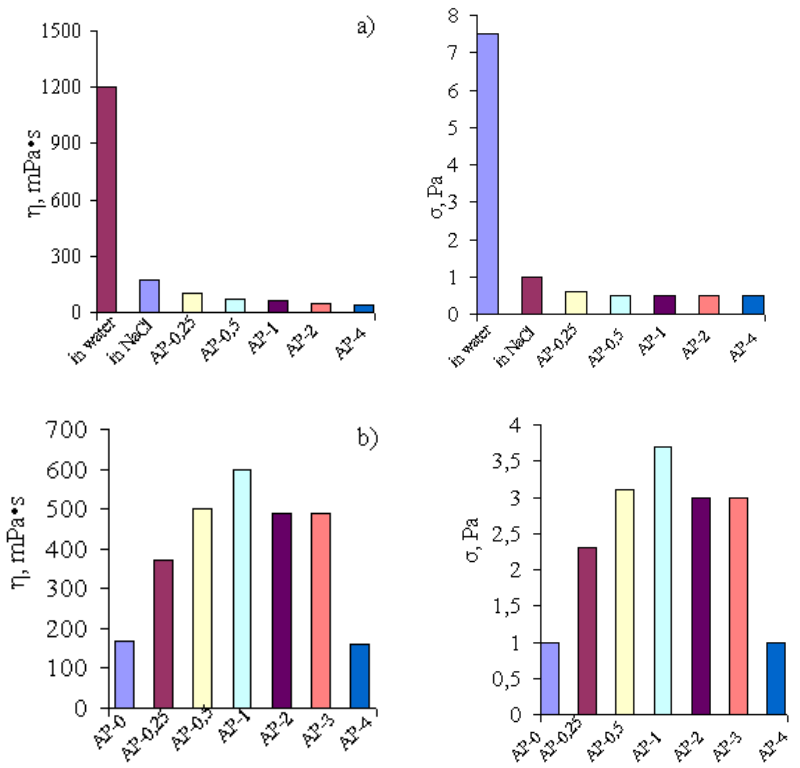

Figure 9. Dynamic viscosity $(\eta)$ and shear stress $(\sigma)$ in (a) aqueous and (b) water-salt solutions of $\operatorname{Pr}-611(1 \% \mathrm{NaCl})$ upon addition of the AP sol. The concentration of $\operatorname{Pr}-611 \mathrm{BC}$ is $0.9 \mathrm{~g} / \mathrm{dl}$. Designations AP-O-AP-4 correspond to the ratio $\left[\operatorname{Pr}_{611 \mathrm{BC}}: \mathrm{Al}^{3+}\right]$ in moles 
The pattern of flow curves points to the Newtonian flow of polymer fluids; an increase in the amount of the added AP sol is accompanied by further worsening of rheological characteristics.

This behavior of aqueous solutions of Pr-611 is observed for a wide range of polymer concentrations from $0.3 \mathrm{~g} / \mathrm{dl}$ to $1.2 \mathrm{~g} / \mathrm{dl}$. Obviously, the reason behind this phenomenon is the compaction of macromolecular coils with an increase in the concentration of $\mathrm{Cl}^{-}$counterions in solution. Upon addition of the AP sol, the solutions of Pr- 611 in $1 \% \mathrm{NaCl}$ behave in a different way (Figure 9b): the viscosity and shear stress increase up to the equimolar ratio of reagents. During the flow, the elastic p roperties of the poly mer-colloid system become more and more pronounced. This is indicative of the formation of a network of interchain bonds due to the crosslinking of macromolecules by gel particles. With an increase in the concentration of the polymer in solution, in the flow of the polymer fluid in the annular gap of the rheometer cell, normal shear stres ses arise in the fluid, and as a result, the Weiss enberg effect appears.

The difference in the behavior of aqueous and water-salt solutions of weakly cationic Pr-611 is apparently associated with the above-mentioned effect of $\mathrm{Cl}^{-}$counterions. In an aqueous solution, the dissociation of functional groups of the polymer leads to the appearance of a positive charge on a macromolecular chain that hinders its interaction with the positively charged APs. In a saline solution, the dis sociation of cationic groups is suppressed; as a result, favorable conditions for appearance of other noncovalent interactions between polymer macromolecules and APs are created. However, the volume fraction of macromolecular coils should be sufficiently high and gels are formed at a Pr-611 concentration of $0.6 \mathrm{~g} / \mathrm{d}$ l or above.

A quite different behavior is observed in the case of solutions of low-anionic Pr-2500. As is known, among noncovalent interactions, electrostatic interactions are the strongest and longest range.[17],[45] In this case, these interactions turn out to be do minant, and as was noted in[30], an increase in the content of anionic groups in the polymer chain brings about the formation of insoluble stoichio metric polycomplexes.

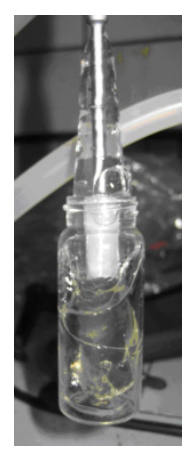

Figure 10. The picture of the Weissenberg effect arising under the action of shear stress in the gels of polyelectrolyte crosslinked by aluminoxane particles

In this case, the interaction of sol particles with Pr-2500 macromolecules is so pronounced that even at a polymer concentration of $0.1 \mathrm{~g} / \mathrm{dl}$ and a minimum AP:Pr-2500 unit ratio of $0.1: 1(\mathrm{~mol} / \mathrm{mol})$, a transparent gel appears in the polymer-collo id solution, whose rheological characteristics cannot be measured by rotational rheometry because of the Weis senberg effect (Figure 10).

Gels, which appear in this case, relate to physical gels. In contrast to the chemically crosslinked gels, they are distinguished by the unstable nature of the network of physical bonds that brings about an uncertainty in the description of their properties. For such gels, determination of the gel point or of the value $m_{c}$ presents a problem. [46] However, for a comparative evaluation of the strength of the physical network in such gels, the yield stress or shear stress, at which the destruction of physical bonds occurs and the material becomes fluid as a liquid, may be used. The tests were conducted on a Yield Rheometer-1 special-purpose instrument (Brookfield) without preliminary shear. The shear rate was varied for diffe rent systems within the interval from 1.5 to $4 \mathrm{rpm}$. Various vane spindles (V-71-73) were applied in the experiments. Figure 11 demonstrates typical flow curves for the gels of polycomplexes of APs with (a) Pr-2500 and (b) Pr-611.
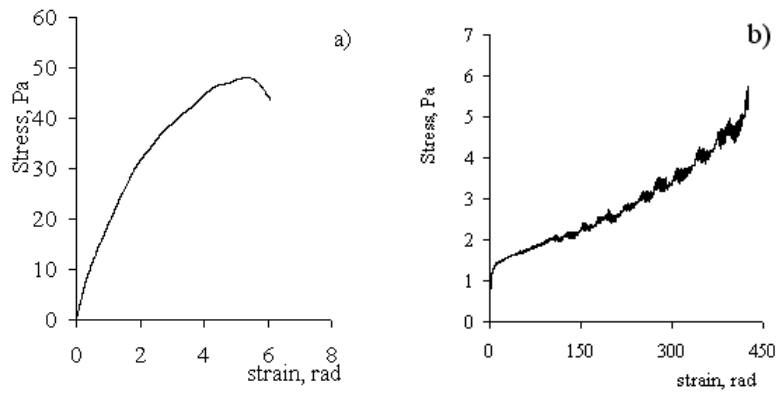

Figure 11. Dependence of shear stress on the deformation of gel based on (a) Pr-2500 and (b) Pr-611. The concentrat ion of copolymers in water-salt solution is $1.2 \mathrm{~g} / \mathrm{dl}$. The ratio of copolymer unit: $\mathrm{Al}^{3+}=2: 1(\mathrm{~mol} / \mathrm{mol})$. The shear rates are $1.5 \mathrm{rpm}$ and $3.0 \mathrm{rpm}$ for $\operatorname{Pr}-2500$ and $\operatorname{Pr}-611$, respectively

The gels based on PAA featured a very small strength; hence, we failed to register this parameter

These data are in agreement with the rheometric results of rotational viscometry measurements; that is, the gels based on Pr-2500 significantly surpass AP gels with Pr-611 with respect to strength. The yield stress, as expected, depends on the concentration of the polymer in solution and on the amount of the AP sol, that is, on the density of the network of intermolecular cross links (Table 2).

Table 2. Yield stresses for gels of copolymers crosslinked by APs

\begin{tabular}{|c|c|c|c|}
\hline Copolymer & $\begin{array}{l}\text { Concentration, } \\
\mathrm{g} / \mathrm{dl}\end{array}$ & 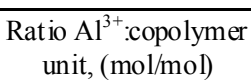 & $\begin{array}{c}\text { Yield } \\
\text { stress, } \mathrm{Pa}\end{array}$ \\
\hline \multirow{8}{*}{ Pr-2500 } & 0.6 & $1: 1$ & 13.3 \\
\hline & 0.9 & $1: 1$ & 29.4 \\
\hline & 1.2 & $0.1: 1$ & 13.3 \\
\hline & 1.2 & $0.5: 1$ & 43.5 \\
\hline & 1.2 & $1: 1$ & 77.2 \\
\hline & 1.2 & $2: 1$ & 75.0 \\
\hline & 1.2 & $4: 1$ & 26.8 \\
\hline & 1.5 & $2: 1$ & 118 \\
\hline Pr-611 & 0.9 & $2: 1$ & 5.5 \\
\hline
\end{tabular}


As is seen from these data, the gels based on Pr-2500 are considerably stronger and more rigid than Pr-611-based gels. If for the former gels, deformation up to the yield stress is small (2-6 rad), then deformation for the Pr-611-based gels it is significantly higher (450 rad). Another specific feature of the Pr-2500 gels with APs is that they are sensitive to the composition of the polycomplex. Figure 12 shows that an increase in the content of APs evidently leads to a gain in the density of the network of interjunction bonds accompanied by strengthening of the gel and detachment of its whole mass fro m cell walls. Moreover, the appearing Weis senberg effect (Figure 10) makes it impossible to achieve the shear stress corresponding to the yield stress (Figure 12, curves 3 and 4).

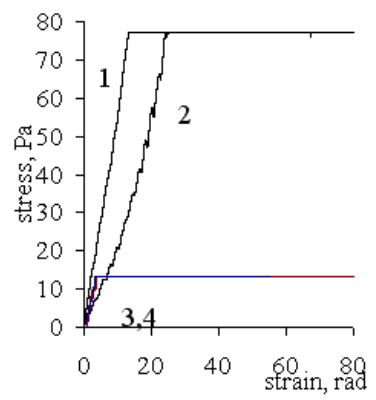

Figure 12. Dependence of shear stress on the strain of gel based on $\operatorname{Pr}-2500$ at different ratios $\operatorname{Pr}-2500$ unit: $\mathrm{Al}^{3+}$ (mol/mol): (1) 1:0.1, (2) 1:0.5, (3) $1: 1$, and (4) $4: 1$. The shear rate is $1.5 \mathrm{rpm}$

In the case of similar rheological systems, the method of dynamic mechanical analys is with the use of a micro-Fourier rheometer may be useful. For a fluid medium containing a polymer solution and colloid particles, both viscous and elastic reactions to the shear load are typical. The value of phase angle $(\alpha)$ between the shear stress and strain for viscoelastic liquids should vary from $\pi / 2$ (the viscous medium) to 0 (the elastic medium). For the gels under consideration, the viscoelastic deformation is evidently determined by two parameters: polymer concentration and ratio between cross linking agents (AP) and polymer. a)

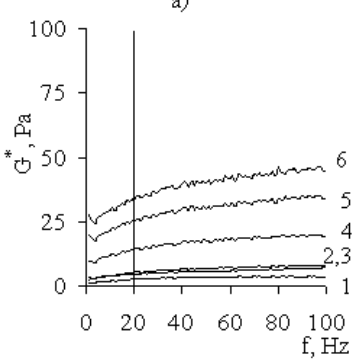

b)

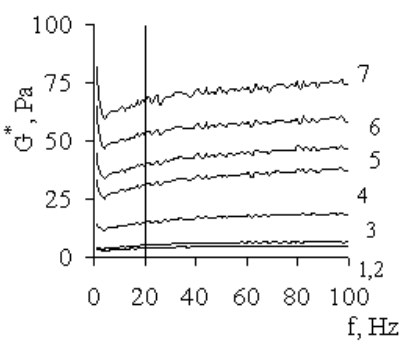

Figure 13. Dependence of complex modulus $\left(G^{*}\right)$ on frequency (f) for water-salt solutions of (a) $\mathrm{Pr}-2500$ and (b) its PCC at polymer concentrations of (1) 0.3 , (2) 0.6 , (3) 0.9 , (4) $1.2,(5) 1.5$, (6) 1.8 , and (7) 2.0

Figure 13 demonstrates variation in the complex shear modulus $\left(\mathrm{G}^{*}\right)$ as a function of the oscillation frequency for the solution of Pr-2500 and the gel of its polycomplex at various polymer concentrations.
As follows from these data, the viscoelastic characteristics of solutions grow with an increase in the strain rate throughout the concentration range from 0.3 to $2.0 \mathrm{~g} / \mathrm{dl}$. In this case, the absolute value of $\mathrm{G}^{*}$ for polycomplexes is always significantly higher than that for the corresponding initial copolymer. The structuring is accompanied by an increase in the elastic component of shear modulus $\left(G^{\prime}\right)$ (Figure 14) and exerts virtually no effect on the loss modulus G” (Figure 15).

Structuring due to the cross linking of poly meric chains of APs is also confirmed by an increase in the storage modulus G' with a rise in the AP:polymer unit. In this case, the viscosity component remains invariable (Figure 16).

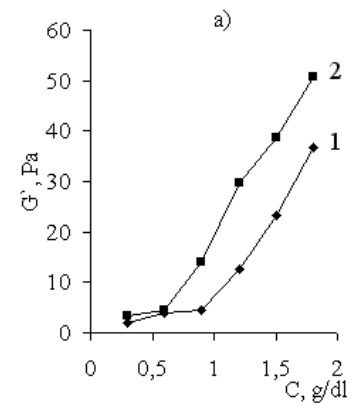

Figure 14. Storage modulus ( $\left.G^{\prime}\right)$ vs. concentration of (1) Pr-2500 and (2) its polycomplex

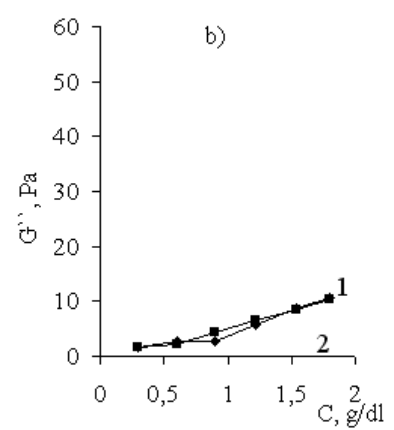

Figure 15. Loss modulus ( $\left.\mathrm{G}^{\prime \prime}\right)$ vs. concentration of (1) Pr-2500 and (2) its polycomplex

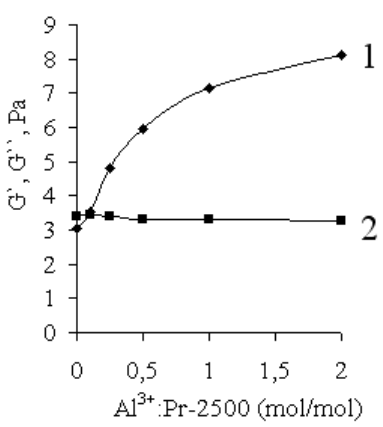

Figure 16. Storage modulus $\left(G^{\prime}\right)$ and loss modulus $\left(G^{\prime \prime}\right)$ vs. AP $\left(\mathrm{Al}^{3+}\right): \operatorname{Pr}-2500(\mathrm{~mol} / \mathrm{mol})$ in the initial mixture. The concentration of Pr-2500 is $0.9 \mathrm{~g} / \mathrm{dl}$

The influence of the amount of the AP sol in the initial mixture on the value of mechanical loss tangent is the specific feature of the system (Figure 17). 
For the polycomplex based on low-basicity $\operatorname{Pr}-2500$, the crosslinking of the poly mer with APs begins at low ratios of reagents.

As was noted above (Table 2), cross linking becomes more pronounced with an increase in this ratio. In this case, the curve exhibits a break in the range $0.5-1.0 \mathrm{~mol}$ of $\mathrm{Al}^{3+}$ per mole of the polymer. A somewhat different behavior is observed for the gels based on a weakly cationic Pr-611.

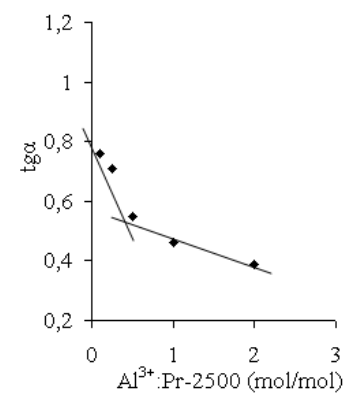

Figure 17. The value of $\operatorname{tg}(\alpha)$ vs. $\mathrm{AP}\left(\mathrm{Al}^{3+}\right): \operatorname{Pr}-2500$ unit molar ratio. The polymer concentration is $0.9 \mathrm{~g} / \mathrm{dl}$

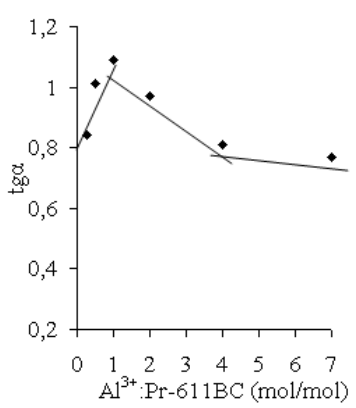

Figure 18. The value of $\operatorname{tg}(\alpha)$ vs. $\mathrm{AP}\left(\mathrm{Al}^{3+}\right): \operatorname{Pr}-611 \mathrm{BC}$ unit molar ratio. The polymer concentration is $0.9 \mathrm{~g} / \mathrm{dl}$

The dependence of $\operatorname{tg}(\alpha)$ on the ratio of reagents shows a complicated pattern (Figure 8). At small a mounts of AP, the elasticity of the gels declines; however, at ratios above 1:1, the elastic reaction increases abruptly to $4: 1$, and then its monotonic growth persists. This specific feature of cationic polyelectrolyte was also noted during the rotational viscometry experiments (Figure 9). This phenomenon is associated with the competition of processes in the compaction of Pr-611 macromo lecules at low concentrations of AP as well as with the predominance of crosslinking processes at higher concentrations of the polymer and a high amount of AP. To provide effective contact of APs with polymer macromolecules, a considerable volume fraction of the polymer in the water-salt solution and a sufficient amount of the crosslinking agent (AP) are evidently required.

\section{Conclusions}

Aluminoxane nanosized particles form stable bonds with polymer chains due to noncovalent interactions that lead to the formation of an extended three-dimensional network rather than form another stable phase in a mixture with aqueous solutions of polyelectrolytes. Aluminoxane nanosized particles in the sol PAHC carry out a part of polyelectrolytes in the initial mixture with acrylamide copolymers and cause the compacting of coils of macromolecules. At that time, noncovalent interactions of APs with units of macro molecules result in their crosslinking. A domination that or another process depends on the type of ionic groups and the presence of a low-molecular-mass indifferent electrolyte $(\mathrm{NaCl})$. As a result, "soft" nanocomposite materials appear in the form of optically transparent gels, in which organic and inorganic components interact at the molecular level. Such organo-inorganic hydrogels lack their own shape, and their yield stress is determined by the nature of the three-dimensional network that appears through the interactions of positively charged alu mino xane clusters with the functional groups of polymers. Structurization takes place under the conditions of a single-phase state of the disperse system stabilized by stable hydrogen bonds in an aqueous solution.

Formed hybrid hydrogels possess viscoelastic characteristics and under the action of shear stress show the "Weissenberg effect." The ratio storage modulus - loss modulus depends on the amount of the AP sol and its ratio to the quantity of the copolymer in the initial mixture. Inorganic aluminoxane particles under consideration are structurally similar to silica partic les formed during the sol-gel synthes is from tetra methoxysilane; however, they differ in the sign of the surface charge, which is negative for silica particles and positive for alu mino xane partic les.

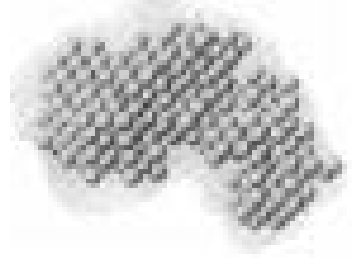

a)

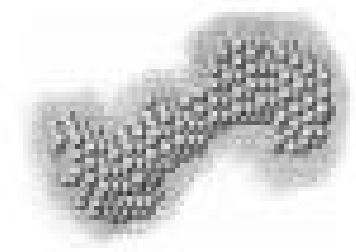

b)
Figure 19. Scattering particles in sol: a) silica $(\mathrm{rd}=4-6 \mathrm{~nm}, \mathrm{df}=2.5)$ [47]; b) aluminoxane particles $(\mathrm{rd}=2.2 \mathrm{~nm}$ and $\mathrm{df}=1.0)[28]$

With due account for this similarity and results of[47], a similar scheme for the spatial structure of hybrid hydrogels may be suggested

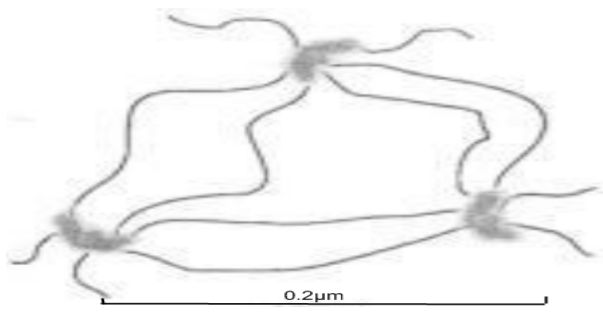

In such a scheme, aluminoxane particles play the role of network junctions that bind polyelectrolyte macro molecules into a common spatial network. It is clear that the density of such a network is determined by the amount of particles, and its strength depends on the nature of noncovalent bonds of particles with polymer chain units, which are the most pronounced for column interactions typical for weakly 
anionic Pr-2500.

\section{REFERENCES}

[1] Richard M. Laine, C. Jeffrey Brinker, Emmanuel P. Giannelis (eds.) "Organic/Inorganic Hybrid Materials", Material Research Society, Warrendale, 2000.

[2] Krishna K. Haldar, Tapasi Sen, Amitava Patra, "Metal Conjugated Semiconductor Hybrid Nanoparticle-Based Fluorescence Resonance Energy Transfer", J. Phys. Chem. C, vol.114, pp.4869-487, 2010.

[3] Vladimir V. Terekhin, Olga V. Dementeva, Viktor M. Rudoy, "Formation Of Ordered Nanoparticle Assemblies By Block Copolymer Lithography Methods", Russ. Chem. Rev., vol.80, pp. 453-472, 2011

[4] Arthi Jayaraman, Kenneth S. Schweizer "Effective Interactions and Self-Assembly of Hybrid Polymer Grafted Nanoparticles in a Homopolymer Matrix", Macromolecules, vol.42, pp. 8423-8434, 2009.

[5] Claudiu B. Bucur, Zhijie Sui, Joseph B. Schlenoff, "Ideal Mixing in Polyelectrolyte Complexes and Multilayers: Entropy Driven Assembly", Amer. Chem. Soc., vol.128, pp. 13690-13691, 2006.

[6] Camila Alves de Rezende, Lay-Theng Lee, Fernando Galembeck, "Silica Nanoparticles at Interfaces Modulated by Amphiphilic Polymer and Surfactant", Langmuir, vol.24, pp. 7346-7353, 2008.

[7] Bruno Alonso, Franck Fayon, Dominique Massiot (eds.) "Hybrid Organic-Inorganic Mesostructured Membranes: Interfaces and Organization at Different Length Scales", J. Phys. Chem. C, vol.114, pp. 11730-11740, 2010.

[8] Takami Akagi, Kazuki Watanabe, Hyungin Kim, Mitsuru Akashi, "Stabilization of Polyion Complex Nanoparticles Composed of Poly(amino acid) Using Hydrophobic Interactions", Langmuir, vol.26, pp. 2406-2413, 2010.

[9] Patrick J. Colver, Catheline A. L. Colard, Stefan A. F. Bon, "Multilayered Nanocomposite Polymer Colloids Using Emulsion Polymerization Stabilized by Solid Particles", J. Amer. Chem. Soc., vol.130, pp. 16850-16851, 2008.

[10] Yuriy Román -Leshkov, Manuel Moliner, Mark E. Davis, "Hybrid Organic-Inorganic Solids That Show Shape Selectivity”, Chem. Mater., vol.22, pp. 2646-2652, 2010.

[11] Bindushree Radhakrishnan, Rajesh Ranjan, William J. Brittain, "Surface Initiated Polymerizations From Silica Nanoparticles” Soft Mater., vol.2, pp. 386-396, 2006.

[12] Sergey S. Ivanchev, Saul Ya. Khaikin (eds.), "Preparation Of Nanocomposites By Alkoxysilane Hydrolysis In A Polypropylene Matrix" Polymer. Sci. Ser.A., vol.44, pp. 996-1001, 2002.

[13] Anatolii D. Pomogailo, Aleksandr S. Rosenberg, Gul'zhian I. Dzhazdinmaieva, "Thermolysis Of Metallopolymers And Their Precursors As A Method For The Preparation Of Nanocomposites" Russ. Chem. Rev., vol.80, pp. 257-291, 2011.

[14] Vladimir V. Vinogradof, AleksandrV. Agafonov, Aleksandr
V. Vinogradov, "Sol-Gel Synthesis Of Nanostructured Materials Based On Aluminum Oxide With Preset Texture Properties", Protection of Metals and Physical Chemistry of Surfaces, vol.46, pp. 582-586, 2010.

[15] John C. Heckel, Lydia M. Kisley, Joseph M. Mannion, George Chumanov, "Synthesis and Self-Assembly of Polymer and Polymer-Coated Ag Nanoparticles by the Reprecipitation of Binary Mixtures of Polymers", Lan gmuir, vol.25, pp. 9671-9676, 2009.

[16] Marián Sedlák , Cestmír Kon ák, “A New Approach to Polymer Self-assembly into Stable Nanoparticles: Poly(ethylacrylic acid) Homopolymers", Macromolecules, vol.2, pp. 7430-7438, 2009.

[17] Immanuel Willerich, Franziska Gröhn, "Molecular Structure Encodes Nanoscale Assemblies: Understanding Driving Forces in Electrostatic Self-Assembly", J. Am. Chem. Soc., vol.133, pp. 20341-20356, 2011.

[18] Ivan M. Papisov, Klaudia.I. Bolyachevskaya, Andrey.A. Litmanovich (eds.), "Structural Effects In Matrix Polycondensation Of Silisic Acid", Eur. Polym. J., vol.35, pp. 2087-2094,. 1999.

[19] Hideharu Mori, Axel H. E. Müller, Joachim E. Klee, "Intelligent Colloidal Hybrids via Reversible pH-Induced Complexation of Poly electrolyte and Silica Nanoparticles", J. Am. Chem. Soc., vol.125, pp. 3712-3713, 2003.

[20] Yang-Yen Yu, Wen-Chang Chen, "Transparent Organic-Inorganic Hybrid Thin Films Prepared From Acry lic Polymer And Aqueous Monodispersed Colloidal Silica", Mater. Chem. and Phys., vol.82, pp. 388-395, 2003.

[21] Ivan M. Papisov, Andrey A. Litmanovich, "On Recognition Phenomena In Polymer-Minute Particle Interactions And Pseudo-Matrix Processes", Coll. and Surf., vol.151, pp. 399-408, 1999.

[22] Jean-Francois Berret, "Stoichiometry of Electrostatic Complexes Determined by Light Scattering", Macromolecules, vol.40, pp. 4260-4266, 2007.

[23] Elmar Poselt, Steffen Fischer, Stephan Foerster, Horst Weller, "Highly Stable Biocompatible Inorganic Nanoparticles by Self-Assembly of Triblock-Copolymer Ligands", Lan gmuir, vol.25, pp. 13906-13913, 2009.

[24] Ivan A. Novakov, Philipp S. Radchenko, Ivan M. Papisov, "Formation of Polycomplexes Based Polyacrylamide and Aluminum Salts", Polymer Science Ser. A, vol.45, pp. 805-808, 2003.

[25] Ivan A. Novakov, Philip S. Radchenko, Andrey S. Pastukhov, Ivan M. Papisov, "The Properties Of Aqueous Solutions Of Polymer-Colloid Complexes Of Polyacrylamide With Poly (Aluminum Hydroxychloride)", Polymer Science, Ser. A, vol.47. pp. 57-60, 2005.

[26] Ivan A. Novakov, Philipp S. Radchenko, Ivan M. Pap isov, "A Study Of The Composition Of Poly acry lamide-Poly aluminu m Chloride Polymer-Colloid Complexes", Polymer Science, Ser. B,. vol.49. pp. 111-113, 2007

[27] Svetlana O. Zakharchenko, Evgeniay A. Litmanovich, Philipp S. Radchenko, (eds.), "Photon Correlation Spectroscopic Study Of The Aggregative Stability Of Colloidal Particles Of Aluminum Pentahydroxide Chloride", Colloid Journal, vol.68, pp. 425-429, 2006. 
[28] Aleksandr S. Ozerin, Philipp S Radchenko, Galina I. Timofeeva, Ivan A.Novakov, "A Study Of Structural And Molecular Weight Characteristics Of Poly(Aluminum Hydroxychloride) Nanoparticles By Small-Angle X-Ray Scattering And Sedimentation Analysis", Nanotechnologies in Russia, vol.4.pp. 93-101, 2009.

[29] Stanislav S. Radchenko, Ivan A. Novakov, Philipp S. Radchenko, Le Van Cong, (eds.), "Interaction of aluminoxane particles with weakly charged cationic poly electrolyte", J. Appl. Polym. Sci., vol.121, pp. 475-482, 2011.

[30] Ivan A. Novakov, Philipp S. Radchenko, Aleksandr S. Ozerin, ElenaV. Rybakova, Stanislav S Radchenko, "Formation Of Polymer-Colloid Complexes Of Aluminoxane Particles With Poly(Acry lic Acid) And Its Copolymers With Acrylamide", Coll. Polym. Sci., vol.289, pp. 1197-1203, 2011.

[31] Ivan A. Novakov, Stanislav S. Radchenko, Philipp S. Radchenko, "Water-Soluble Polymer-Colloid Complexes Of Aluminum Polyhydroxochloride And Polyacrylamide In Separation Of Model And Real Dispersions", Russian Journal of Applied Chemistry, vol.77, pp. 1699-1705, 2004.

[32] Stanislav S. Radchenko, Ivan A. Novakov, Philipp S. Radchenko, Elena V.Rybakova, Patent 2288182, RF, 2006.

[33] Stanislav S. Radchenko, Ivan A. Novakov, Philipp S. Radchenko, Le Van Cong, Elena V. Rybakova, "Flocculating Properties of Water-Soluble Polymer-Colloid Complexes of Aluminoxane Particles with Weakly Charged Cationic Polyelectrolytes", J. of Water Res. and Prot., vol.3, pp. 213-221, 2011.

[34] Stanislav S. Radchenko, Ivan A. Novakov, Philipp S. Radchenko, Aleksandr S. Ozerin, Pavel S. Zeltser, Sergey U. Jakubovski, Patent 2348792, RF, 2009.

[35] Ivan A. Novakov, Stanislav S. Radchenko, Philipp S. Radchenko, Aleksandr S. Ozerin, Aleksey B. Karaulov, "Poly acry lamide-Aluminum Pentahy droxochloride-Urea Formulations As Waterproofing Agents For Oil Pool", Russian Journal of Applied Chemistry, vol.81, pp. 1389-1394, 2008.

[36] Nikolay A. Kidalov Philipp S. Radchenko Viktor F. Zakutaev, Ivan A. Shamrey Stanislav S. Radchenko Patent 2449854 RF, 2012.
[37] Gebhard Schramm, "A Practical Approach to Rheology and Rhometry”, Gebrueder HAAKE GmbH, Karlsrue, 1994.

[38] Ivan A. Novakov, Philipp S. Radchenko, Aleksandr S. Ozerin, Elena V. Rybakova, "Interaction Of Aluminum Polyhy droxochloride Sol And Poly(4-Viny lbenzene Sulfonic Acid) Sodium Salt", Polymer Sci. A., V.53, pp. 364-368, 2011.

[39] Nikolay U. Bikadorov, Stanislav S. Radchenko, Ivan A. Novakov, Philipp S. Radchenko, Ol'ga K. Gohova, Patent 2210539, RF, 2005.

[40] Stanislav S. Radchenko, Nikolay U. Bikadorov, Ivan A. Novakov, Ol'ga K. Gohova, (eds.), "Structuring In Concentrated Solutions Of High-Basicity Aluminum Hydroxy chloride And In New Coagulant Formulations On Its Base”, Russian Journal of Applied Chemistry, vol.75. pp. 529-534, 2002

[41] Michael Rubinstein., Ralph H. Colby, "Polymer Physics", Oxford, N.-Y., Oxford Univer. Press, 2003.

[42] Aleksandr Y. Grosberg, Aleksey R. Khokhlov, "Statistical Physics of Macromolecules”, N-Y, AJP Press, 1994.

[43] Michael J. Gidley, "Molecular Mechanisms Underlying Amylose Aggregation And Gelation”, Macromolecules, vol.22, pp. 351-358, 1989.

[44] Aleksey S. Makarov, Irina A. Andreeva, Viktor Yu. Tretinnik, "Rheological Properties of Polymer-Containing Aqueous Aerosil Dispersions", Colloid Jornal, vol.63, pp. 731-737, 2001.

[45] Nikolai V. Churaev, "Surface Forces And Physicochemistry Of Surface Phenomena”, Russ. Chem. Rev., vol.73, pp. 25-42, 2004.

[46] Klaas Te Nijenhuis, H. Henning Winter, "Mechanical Properties At The Gel Point Of A Crystallizing Poly(Vinyl Chloride) Solution", Macromolecules, vol.22. pp. 411-414, 1989.

[47] Irina V. Bakeeva, Ludmila A. Ozerina, Aleksandr N. Ozerin, Vitalii P. Zubov, "Structure And Characteristics Of Organic-Inorganic Hybrid Hydrogels Based On Poly(N-Vinylcaprolactam)-SiO ${ }_{2}$ ", Polymer Science Ser. A, vol.52, pp. 496-505, 2010. 